\title{
Regional Distribution of Pulmonary Ventilation and Perfusion in Obesity *
}

\author{
H. S. Holley, $\dagger$ J. Milic-Emili, M. R. Becklake, and D. V. Bates \\ (From the Joint Cardiorespiratory Service, McGill University Clinic, Royal Victoria Hospital, \\ Montreal, Canada)
}

\begin{abstract}
Summary. Five women and three men, all obese and weighing 95 to $140 \mathrm{~kg}$, were studied by routine pulmonary function tests and by a radioactive xenon technique, while seated upright at rest, to measure the regional ventilation and perfusion distribution in the lung.

In four subjects in whom the expiratory reserve volume averaged $49 \%$ of predicted normal, the ventilation distribution as measured with ${ }^{133}$ xenon was normal. In the remaining four subjects, in whom the expiratory reserve volume was reduced to less than $0.4 \mathrm{~L}$ and averaged only $21 \%$ of predicted values, the distribution of a normal tidal breath was predominantly to the upper zones.

In all subjects the perfusion distribution was predominantly to the lower lung zones but was slightly more uniform than in normal nonobese subjects. During tidal-volume breathing, therefore, in four subjects the ventilation and perfusion distribution was substantially normal, whereas in the remaining four perfusion was maximal in the lower zones, to which ventilation was significantly reduced.

These findings show that there may be significant ventilation/perfusion abnormality on a regional basis in obese subjects, this abnormality bearing a close relationship to the reduction in expiratory reserve volume, a finding predictable from recently published data on normal nonobese subjects (1). The abnormalities of ventilation/perfusion relationships that were demonstrated in four of the eight obese subjects could cause a reduction in arterial oxygen tension during resting tidal ventilation.
\end{abstract}

\section{Introduction}

The term "Pickwickian syndrome" was introduced by Burwell, Robin, Whaley, and Bickelmann (2) to describe a patient with alveolar hypoventilation, polycythemia, and right heart failure. As the patient lost weight, his pulmonary and

\footnotetext{
* Submitted for publication July 15, 1966; accepted December 1, 1966.

Supported in part by grants from the John A. Hartford Foundation and the Medical Research Council of Canada.

†This material formed the basis of Dr. Holley's thesis, accepted by McGill University, Montreal, for the degree of Master of Science, 1965.

$\ddagger$ Address requests for reprints to Dr. D. V. Bates, Royal Victoria Hospital, Pine Ave. W., Montreal 2, Canada.
}

cardiac status returned to normal, suggesting that the alveolar hypoventilation was not attributable to any structural pulmonary disease but rather was a functional consequence of excessive weight. On the assumption that the fully developed syndrome represented the end result of such functional abnormalities operating over a period of time, Said (3) set out to elucidate the sequence of events in obesity that leads to the development of the Pickwickian syndrome. In a study of patients selected solely because they were overweight, he noted that although hypoxemia (as evidenced by a reduced arterial $\mathrm{O}_{2}$ tension) was present in most subjects, it was not always accompanied by hypoventilation (as evidenced by an increase in arterial $\mathrm{CO}_{2}$ tension). He concluded that disturb- 


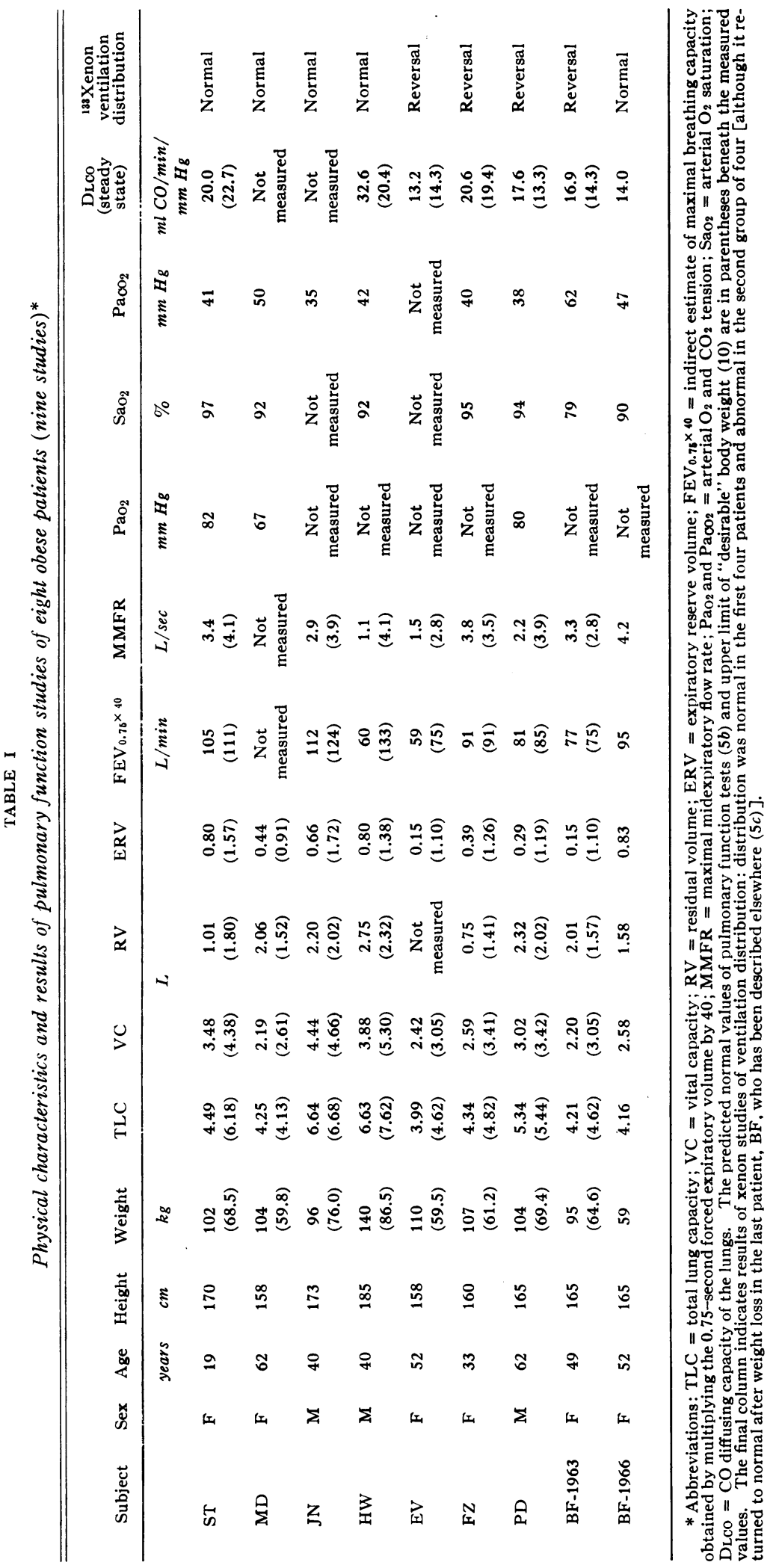


ance of regional ventilation/perfusion relationships in the lung must be a common underlying feature.

Recent studies in our laboratory have shown that in normal subjects seated upright, ventilation to the lower zones may be seriously impaired when the subject breathes at low lung volumes (i.e., near residual volume), since these zones begin to fill preferentially only when functional residual capacity (FRC) is approached $(1,4)$. Since the expiratory reserve volume (ERV) is commonly reduced in obesity ( $5 a)$, it follows that the fat person breathes closer to residual volume (RV) than does the nonobese person; this, together with the fact that the obese subject's tidal volume often is small $(2,3)$, might result in reduced ventilation to the lower lung zones. Perfusion, on the other hand, presumably would remain gravity oriented (6-9). Thus, lower lung zones of obese subjects might have considerably reduced ventilation in relation to their perfusion.

To test this hypothesis, we measured the regional distribution of ventilation and perfusion in eight subjects selected on the basis of their being overweight.

\section{Methods}

The physical characteristics and pulmonary function data for the eight subjects are shown in Table I. Their ages ranged from 19 to 62 years; five were women. In every case body weight was more than $20 \mathrm{~kg}$ above the desirable weight predicted on the basis of sex and height, and in several (MD, HW, EV, and FZ) it was more than $40 \mathrm{~kg}$ above predicted. The vital capacity (VC) was reduced in most of the patients, and the ERV was markedly decreased in all (both measurements were made with the patients in the sitting position). Considerable reduction in the ventilatory flow rates occurred in one subject (HW), who had a history of asthma. Some degree of arterial unsaturation was recorded in three of the six subjects in whom arterial blood samples were analyzed. The arterial $\mathrm{PcO}_{2}$ was elevated in one patient who was considered to have some degree of hypoventilation (MD), and considerably elevated in another (BF-1963) who had been studied over a 5-year period and was known to be a case of the Pickwickian syndrome $(5 c)$. The steady state CO-diffusing capacity was normal in all six subjects in whom it was measured. None of the patients had any clinical evidence of congestive heart failure at the time of investigation, and none was thought to have any significant degree of pulmonary infection when the xenon studies were performed.

The equipment used during the ${ }^{133}$ xenon studies has been described previously $(1,6)$. In this study, eight or ten scintillation counters, fitted with cylindrical lead collimators $17.6 \mathrm{~cm}$ long, were positioned behind both lungs at various levels in relation to individual chest radiographs. The regional distribution of ventilation was studied in two ways. a) Starting from FRC, the subject inspired a tidal-volume breath of a mixture of ${ }^{133}$ xenon and air and at the end of the inspiration held it for a few seconds with glottis open until the scintillation counters recorded stable count rates. b) Starting from various lung volumes ranging from 0 to $80 \% \mathrm{VC}$, the subject inspired the same mixture to total lung capacity (TLC) and held his breath until stable rates had been recorded. Each subject made eight to ten such maximal inspirations initiated from various lung volumes. After each maneuver, the subject hyperventilated for a few minutes to clear ${ }^{133}$ xenon rapidly from the lungs.

Regional count rates after each single inspiration of ${ }^{133}$ xenon depend on the relative amounts of the gas that have reached the various lung regions in relation to lung volume and on the absorption characteristics of tissues underlying each counter. To allow for these latter differences in geometry, each subject rebreathed from the closed-circuit spirometer system containing a known concentration of ${ }^{133}$ xenon in air, as previously described (6). At equilibration, the ratio of lung concentration to external count rate was obtained for each counter at the appropriate level of lung volume studied. From these measurements the regional concentrations of ${ }^{133}$ xenon could be computed, as described fully elsewhere (1). In brief, it has been shown that the gas dilution ratio at the end of maximal inspirations initiated from various overall lung volumes can be used to compute the corresponding regional preinspiratory volumes. In this way, the proportionate expansion of various parts of the lung may be computed.

The regional distribution of perfusion was studied by injecting $1 \mathrm{mc}{ }^{133}$ xenon dissolved in $5 \mathrm{ml}$ saline into an antecubital vein. Approximately $95 \%$ of the amount injected diffuses into the alveolar gas during its first passage through the lung; hence the resulting concentration in each region is proportional to the regional pulmonary blood flow per unit gas volume. As the amount of ${ }^{133}$ xenon inhaled or injected may vary, it is difficult to compare regional concentrations of ${ }^{133}$ xenon obtained in different studies. Accordingly, the results obtained during tidal-volume inspiration and during injection have been expressed as distribution indexes as described by Ball, Stewart, Newsham, and Bates (6). In these indexes, the regional concentration of ${ }^{138}$ xenon is expressed as a percentage of the concentration that would have existed had the xenon administered been distributed evenly throughout the lung.

All of the xenon studies were performed with the subject seated comfortably upright, fully supported in a chair, after 15 to 20 minutes' rest. All restrictive garments were removed before the test.

\section{Results}

Figure 1 illustrates the distribution of ventilation and perfusion in the eight subjects studied. 

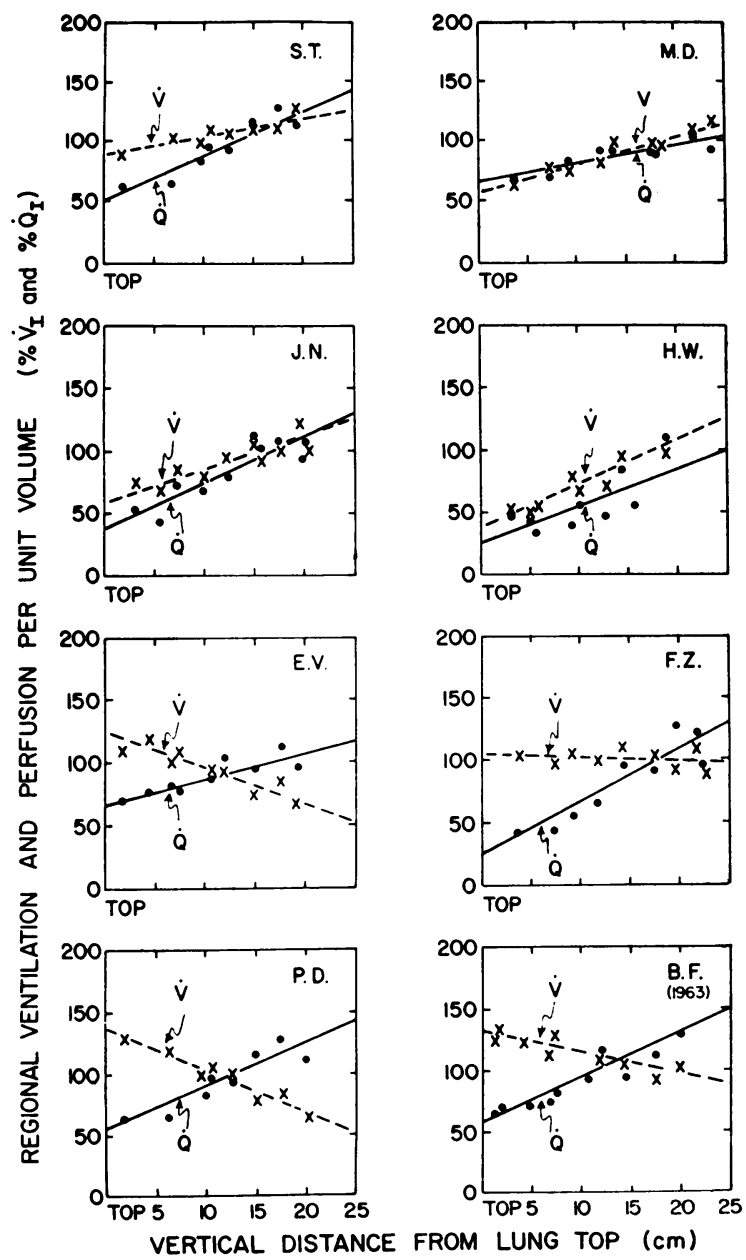

Fig. 1. Results of ventilation and perfusion disTRIBUtion STUdies on EIGHT OBESE subjects. All studies were done during normal tidal-volume breathing with the subject seated upright. Ventilation (V) and perfusion ( $\dot{Q})$ are expressed in terms of distribution indexes; if ventilation and perfusion per unit lung volume had been uniform throughout the lung, the indexes would have been 100 in all regions. The regression lines were calculated statistically. In the upper four subjects, $V$ was greater in the (gravitationally) lower zone of the lung than in the upper zone. In the lower four subjects, however, ventilation distribution was predominantly to the upper zones. $\dot{Q}$ distribution was in the same direction in all subjects.

In this and subsequent Figures the subjects are identified by their initials and appear in the same order. Ventilation and perfusion distribution indexes (on the ordinates) are plotted against vertical distance (in centimeters) from the lung top (on the abscissas). The discrete points represent ventilation and perfusion measurements obtained from counters positioned as indicated on the abscissa.

In normal persons the perfusion index increases approximately linearly with vertical distance down the lungs $(8,9)$. A similar pattern was observed in all of the obese subjects (Figure 1). The average values of the slopes of the regression lines and of their intercepts (at distance $=0$ ) were 3.23 and 47.5, respectively. The values for normal subjects reported from this laboratory are 5.8 and 15.0 , respectively (9). Thus, in the obese group as a whole, the distribution of pulmonary blood flow was somewhat more uniform than in nonobese normal subjects.

In Figure 1 is also shown the distribution of ventilation following a tidal-volume inspiration. In the upper four subjects ( $\mathrm{ST}, \mathrm{MD}, \mathrm{JN}, \mathrm{HW}$ ), ventilation increased progressively from the top to the bottom of the lung, in comparable fashion to that which occurs in normal persons (1). In the other subjects (EV, FZ, PD, and BF-1963), however, the distribution of ventilation per unit lung volume was reversed, the upper lung regions being relatively better ventilated than the lower. This reversal of ventilation distribution was striking in three subjects, but in FZ the ventilation distribution was fairly uniform. Even so, this represents an abnormality by contrast with findings in normal persons.

From these data it may be concluded that four subjects had normal ventilation distribution, and in the other four there was reversal of ventilation distribution (these differences are indicated in the Table). In addition, the gradient of perfusion distribution down the lung appeared to be slightly less well marked than in normal subjects, but was in the normal direction.

In Figure 2 the differences in regional lung volume are shown in greater detail. This method of plotting data of regional lung volume (ordinate) against total lung volume (abscissa) has been applied to normal subjects in previous publications from this laboratory $(1,9)$. The actual FRC and $\mathrm{RV}$ in relation to the over-all lung volume are shown at the top margin of each panel. In all subjects, the distribution pattern of ventilation was similar to that which occurs in normal subjects, i.e., at low lung volumes ventilation was predominantly to the upper zones, whereas the opposite was true at high lung volumes $(1,9)$. In four of 
the subjects ( $\mathrm{ST}, \mathrm{MD}, \mathrm{JN}$, and $\mathrm{HW}$ ) the distribution of ventilation above FRC was normal (i.e., preferentially distributed to the lower zones). In the other four (EV, FZ, PD, BF-1963) the distribution of ventilation became preferential to the lower lung zones only some several hundred milliliters above FRC, and indeed it is in these four subjects that we observed a reversal of ventilation distribution (Figure 1). It may be noted that in these four subjects the ERV averaged only $21 \%$ of predicted value (range, 14 to $31 \%$ ), whereas in the other four, who had normal ventilation distribution, ERV averaged $49 \%$ of predicted value (range, 39 to $58 \%$ ), as indicated by the data in the Table.

In a lung not disorganized by disease, it is possible to express the ratio of ventilation/perfusion distribution within the different lung zones as an approximate guide to the probable $V / \dot{Q}$ ratio, recognizing that when ventilation and perfusion are measured as separate functions, as in the present studies, there is bound to be uncertainty as to the resulting ratio. From such data, it is possible to compute regional partial pressures for $\mathrm{O}_{2}$ and $\mathrm{CO}_{2}$, provided that an assumption is made concerning the composition of mixed venous blood and that the influence of the respiratory dead space can be ignored (8). Figure 3 shows such computations made in the present study. In all cases the over-all $\mathrm{V} / \dot{Q}$ ratio was assumed to be 0.8 . In the four subjects with normal ventilation distribution (upper four panels), $V / \dot{Q}$ distribution was more uniform than in normal nonobese seated man (9), mainly as a consequence of the more uniform distribution of perfusion per unit lung volume that occurs in obese persons. Thus, the regional $\mathrm{PO}_{2}$ in the lungs of these subjects will be more uniform than normal, and the decreased arterial $\mathrm{O}_{2}$ saturation that was observed in two ( $\mathrm{HW}$ and MD) cannot be directly attributed to any disturbance in regional $\mathrm{V} / \dot{\mathrm{Q}}$. Most likely it is attributable to hypoventilation, which indeed was present in MD. By contrast, in the four subjects with a reversed distribution of ventilation (lower four panels), the regional differences in $V / \dot{Q}$ were considerably greater than in normal nonobese man. In three cases (EV, PD, and BF-1963) the computed $V / \dot{Q}$ was lower than 0.5 in the lower lung regions, and the regional $\mathrm{Po}_{2}$ values may have approached those of mixed venous blood. In these
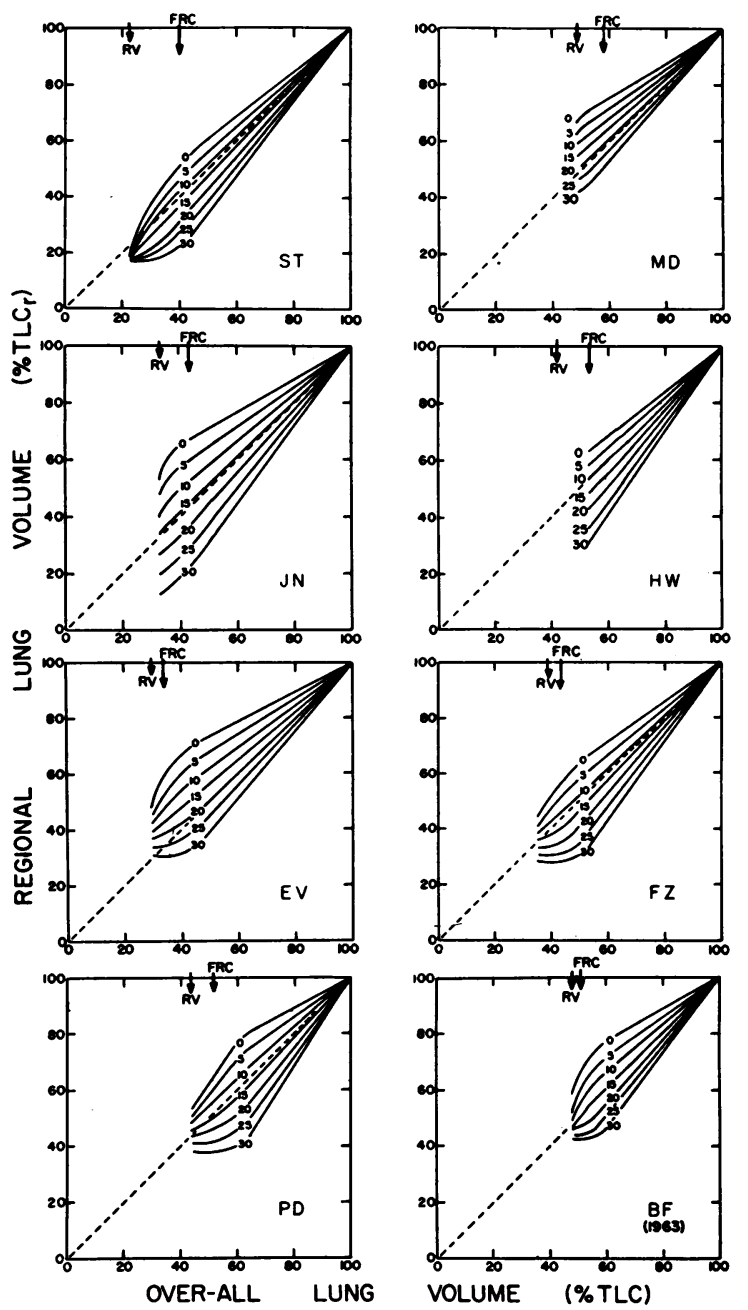

Fig. 2. Regional LUNG volumes in Relation to OVER-ALL LUNG VOLUME IN THE EIGHT OBESE SUBJECTS. The broken lines of identity indicate theoretical values that would obtain if percentile expansion of all regions were uniform. The divergent lines in each rectangle represent data in relation to distance from lung top (0) to lung base $(30 \mathrm{~cm})$; the method of deriving these lines is explained in the text and elsewhere (1). Measured functional residual capacity (FRC) and residual volume (RV) values are shown at the top of each panel. The upper four panels depict the findings in the four obese subjects in whom the pattern of ventilation in the tidalvolume range was normal: the distribution lines become linear at their FRC level, with distribution predominantly to the lower zones, as shown by the steeper curve for the $30-\mathrm{cm}$ lines. In the lower four patients, the FRC level is in the alinear part of the curve, resulting in reduced ventilation distribution to the lower zones of the lung during tidal-volume breathing. Regional and over-all lung volumes are given as per cent of total capacity. 

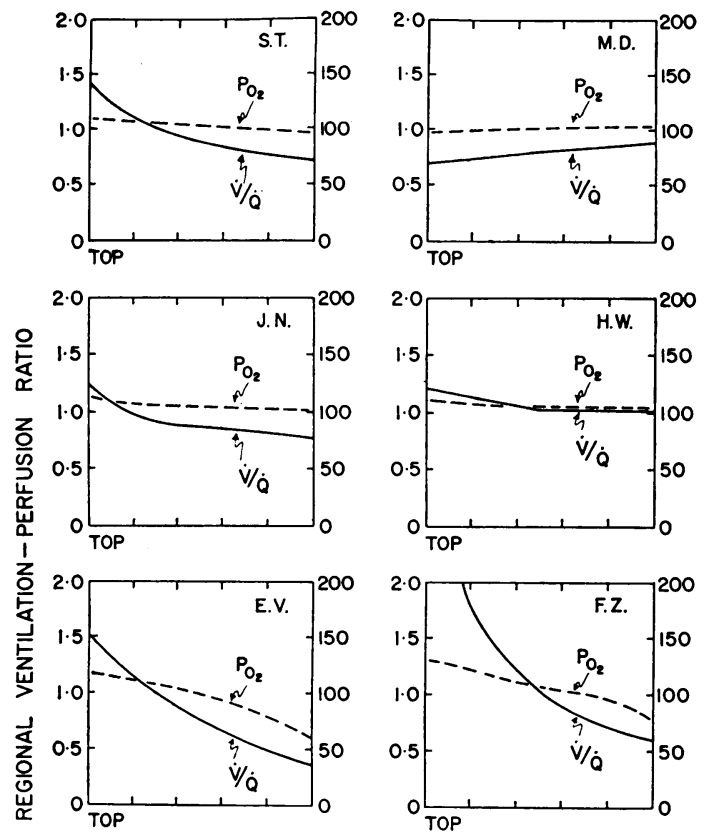

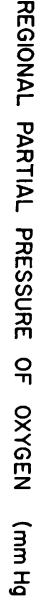
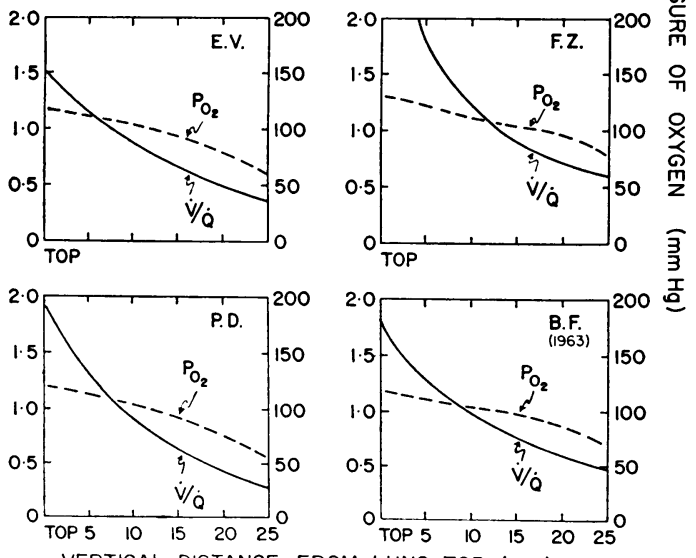

VERTICAL DISTANCE FROM LUNG TOP $(\mathrm{cm})$

Fig. 3. Calculated regional partial pressures of OXYGEN $\left(\mathrm{PO}_{2}\right)$ AND REgIONAL VENTILATION/PERFUSION Ratios in the eight obese subjects. The regional $\mathrm{PO}_{2}$ was calculated by assuming a mixed venous $\mathrm{O}_{2}$ content and using the $\mathrm{O}_{2} / \mathrm{CO}_{2}$ diagram directly (8). The $\mathrm{V} / \dot{Q}$ was computed from knowledge of the (separately measured) ventilation and perfusion distribution. Note that in subjects with ventilation abnormality (the four lower panels) the computed $V / \dot{Q}$ falls to low levels in the dependent regions of the lung and results in low mean $\mathrm{PO}_{2}$ in such regions.

subjects, therefore, the disturbance of the $\mathrm{V} / \dot{Q}$ ratio, a consequence of regional hypoventilation of the lower lung zone during tidal-volume breathing, may well be responsible for arterial hypoxia. In one of these subjects (BF-1963) alveolar hypoventilation was known to be present and was probably an additional factor in the production of the low arterial saturation.

In $\mathrm{BF}$, xenon studies were repeated 3 years later, in 1966. Small-bowel bypass had been constructed in September 1965 to induce weight loss. When restudied, after she had lost $36 \mathrm{~kg}$ body weight, the xenon distribution of ventilation was normal. It is of considerable interest that the measured ERV had increased from $0.15 \mathrm{~L}$ in 1963 to $0.83 \mathrm{~L}$ in 1966 (Table I). This finding lends support to the view that ventilatory changes in the lung are a consequence of reduction in the ERV, and that if, due to weight loss, the ERV is restored to normal, the ventilation distribution pattern will improve.

\section{Discussion}

This study has validated the hypothesis that in obese subjects, seated upright, ventilation to lower lung zones may be impaired, resulting in abnormally low $V / Q$ ratios in these areas. Only four of the eight subjects studied showed this change, which appeared to be more closely related to reduction in the ERV than to the degree of obesity. In the other four subjects, although this $V / \dot{Q}$ disturbance was not demonstrated under the conditions of the test (i.e., seated upright), it might well be present in circumstances that are known to reduce the ERV, e.g., in the supine position, or when wearing supportive abdominal garments, as is usual among the obese. No rigorous conclusions can be drawn concerning the effect of the demonstrated disturbances of the $V / \dot{Q}$ relationships in the lower lung zones on gas exchange and on arterial blood gases: This information could have been obtained only if arterial blood samples had been drawn with the subjects in precisely the same position and at the same time as the xenon studies were made. Indeed, in the obese subject, neither the $V / \dot{Q}$ relationships that obtain in one specific position nor a single arterial blood sample necessarily represents the situation at all times. It is clear from Figure 2 that when an obese subject takes a full inspiration from FRC the lower zones are better ventilated than the upper. Hence, the abnormal $V / \dot{Q}$ distribution pattern largely disappears then, and the arterial blood gas tensions should return toward normal. Such abrupt changes in oxygen saturation following a few deep breaths commonly occur in obese subjects (3); the present data suggest that the phenomenon is somewhat more subtle than can be accounted for simply by an increase in total ventilation per se.

The distribution of perfusion was more nearly uniform from top to bottom of the lung than 
occurs in normal nonobese subjects. This may be due to increased pulmonary perfusion pressure, which in turn might be the consequence of a higher than normal resting cardiac output, though some change in pulmonary vascular resistance cannot be excluded. It seems unlikely that the findings could be explained either by the somewhat shorter stature of these obese patients compared with normal control subjects, or by the fact that they were more apprehensive (which in itself would occasion a slight increase in cardiac output), though these factors cannot be excluded.

It is of particular interest that the reversal of ventilation in a very obese patient $(B F)$, which was present during the initial studies when she weighed $95 \mathrm{~kg}$, was replaced by a normal pattern when her weight had been reduced to $59 \mathrm{~kg}$. The ERV had increased from 0.15 to $0.83 \mathrm{~L}$ over the same period (see Table). A considerable degree of alveolar hypoventilation had been present during the 5 years preceding the surgical operation that led directly to improvement in her condition (5c). Thus, the improvement in the blood gases that had occurred when she was studied 1 year after operation may have been attributable to the improvement in ERV and consequent improvement in ventilation distribution, as well as to a decrease in metabolic demands.

As indicated in Table I, even very large increases in weight are not necessarily accompanied by severe decreases in ERV. The reason for this, possibly, is that in some patients the increase in body mass may occur in such regions as the buttocks, which would not interfere with the ERV, whereas in other patients fat deposition may occur mainly in the abdominal wall and thus cause considerable elevation of intra-abdominal pressure and marked restriction of the ERV. Thus, the impairment of $V / \dot{Q}$ relationships that may occur in obese persons may be critically dependent upon the location of excess body weight. Considerable ventilation reversal was shown in $P D$, although the ventilatory tests were normal and weight gain had not been so great in absolute terms as in some of the other subjects. On the other hand, his ERV was reduced to $0.29 \mathrm{~L}$, compared with a predicted normal value of $1.19 \mathrm{~L}$.

In general terms, abnormality of ventilatory distribution was not observed unless the ERV had fallen below $0.40 \mathrm{~L}$. The two groups shown in
Table I are separated by this criterion-but only just, since MD showed no ventilation reversal even though ERV was $0.44 \mathrm{~L}$, whereas $\mathrm{FZ}$ showed a flattening of ventilation distribution with an ERV of $0.39 \mathrm{~L}$. However, a significant degree of ventilation reversal was noted only in $\mathrm{EV}$, $\mathrm{PD}$, and BF-1963, in all of whom the ERV was less than $0.30 \mathrm{~L}$. Therefore, this ERV value may be taken as an approximate indicator of whether a ventilation distribution defect is likely to be present in any given patient.

\section{Acknowledgments}

The subjects were referred to us through the courtesy of Dr. M. M. Hoffman, Chief of the Endocrine Metabolic Service of the Royal Victoria Hospital.

\section{References}

1. Milic-Emili, J., J. A. M. Henderson, M. B. Dolovich, D. Trop, and K. Kaneko. Regional distribution of inspired gas in the lung. J. appl. Physiol. 1966, 21, 749.

2. Burwell, C. S., E. D. Robin, R. D. Whaley, and A. G. Bickelmann. Extreme obesity associated with alveolar hypoventilation-a Pickwickian syndrome. Amer. J. Med. 1956, 21, 811.

3. Said, S. I. Abnormalities of pulmonary gas exchange in obesity. Ann. intern. Med. 1960, 53, 1121.

4. Dollfuss, R. E., J. Milic-Emili, and D. V. Bates. Regional ventilation of the lung studied with boluses of ${ }^{133}$ xenon. Respiration Physiology. In press.

5. Bates, D. V., and R. V. Christie. Respiratory Function in Disease. An Introduction to the Integrated Study of the Lung. Philadelphia and London, Saunders, $1964, a)$ p. $104, b)$ pp. $92-93, c)$ pp. 372373.

6. Ball, W. C., Jr., P. B. Stewart, L. G. S. Newsham, and D. V. Bates. Regional pulmonary function studied with xenon ${ }^{133}$. J. clin. Invest. 1962, 41, 519.

7. Bryan, A. C., L. G. Bentivoglio, F. Beerel, H. MacLeish, A. Zidulka, and D. V. Bates. Factors affecting regional distribution of ventilation and perfusion in the lung. J. appl. Physiol. 1964, 19, 395.

8. West, J. B., and C. T. Dollery. Distribution of blood flow and ventilation-perfusion ratio in the lung, measured with radioactive $\mathrm{CO}_{2}$. J. appl. Physiol. 1960, 15, 405.

9. Anthonisen, N. R., and J. Milic-Emili. Distribution of pulmonary perfusion in erect man. J. appl. Physiol. 1966, 21, 760.

10. Documenta Geigy, Scientific Tables, 6th ed., K. Diem, Ed. Montreal, Geigy Pharmaceuticals, 1962, p. 624; quoted from Metropolitan Life Insurance Company Statistical Bulletin no. 40, 1959. 